\title{
Electron-Dense Plasma Membrane Layer
}

National Cancer Institute

\section{Source}

National Cancer Institute. Electron-Dense Plasma Membrane Layer. NCI Thesaurus.

Code C32499.

The hydrophilic portions of the plasma membrane as seen during transmission electron

microscopy. This electron density is caused by abundant staining by heavy metal-

containing dyes. 